\title{
El proyecto fenomenológico hegeliano y el rol de la "Autoconciencia" en la identificación absoluta del ser y el pensar
}

\author{
Miguel Alejandro Herszenbaun \\ UBA / CONICET / CIF / GEK \\ herszen@hotmail.com
}

Recibido 8/10/2015

Aprobado 9/08/2016

\section{Resumen}

El presente trabajo analiza la relación entre los objetivos generales de la Fenomenología del espíritu, trazados en su "Introducción", y el capítulo "Autoconciencia". Entendiendo que la Fenomenología tiene como objetivo general alcanzar una identificación entre pensamiento y ser que supere la posición gnoseológica kantiana, sostendremos como hipótesis que el aporte concreto de la "Autoconciencia" a estos objetivos es permitir una primera reunión de los polos objetivo y subjetivo en la experiencia de un objeto que ya no es mero objeto, sino pensamiento: la experiencia que sufre la conciencia en la "Autoconciencia" resulta una primera realización efectiva del pensamiento en el ser, consistente en saberse a sí misma como objeto y conocer los restantes objetos como "yoes" ajenos. Esta experiencia conduce a redefinir la estructura ontológica del objeto de forma tal que el saber se realiza efectivamente en la realidad, bajo la forma de un objeto que es al mismo tiempo saber. Así, se da un paso fundamental hacia el cumplimiento de los objetivos generales de la Fenomenología.

Palabras clave

Autoconciencia - Experiencia - Dialéctica -Fenomenología del espíritu - Hegel 
The phenomenological project of Hegel and the role of the "Self-consciousness" in the absolute identification between being and thinking

\section{Abstract}

This paper intends to analyze the relationship between the main goals of the Phenomenology of spirit, established in its "introduction", and the chapter "Selfconsciousness". The Phenomenology seeks as its general aim an identification between thought and being that may outrange the Kantian epistemological standpoint. I will defend the thesis that the main contribution of "Self-consciousness" to this general aim is to allow a first assemblage of the subjective and objective sides in the experience of an object which is not a mere object but also thought: The experience that the consciousness undergoes in this chapter is a first realization of thought in being, which consists in knowing itself as object and knowing objects as other "I's". This experience leads to redefining the ontological structure of the object by identifying being and thought so thought exists in the reality as an object which is at the same time being. Thus, an important step is made towards the fulfillment of the general aims of the Phenomenology.

\section{Keywords}

Self-consciousness - Experience -Dialectics - Phenomenology of spirit - Hegel

\section{Introducción}

La Fenomenología del espíritu es una obra difícil de abordar por los diversos enfoques que pueden ofrecer una mirada legible sobre el texto. Aquí propondremos una lectura sobre los objetivos y métodos de la Fenomenología desde una perspectiva teorética -atendiendo sus aspectos gnoseológicos y metafísicos- para luego precisar los aportes de la primera sección del capítulo "Autoconciencia" a estos objetivos generales. 


\section{Método y objetivos de la Fenomenología del espíritu}

La originalidad de la Fenomenología del espíritu vuelve al propósito de establecer sus objetivos y método una tarea nada trivial. Su inserción en la tradición idealista ha fomentado en reiteradas oportunidades su comparación con la obra kantiana ${ }^{1}$. Sin desestimar esta comparación, es de fundamental interés reconocer sus particularidades a fin de comprender el proyecto fenomenológico en su originalidad.

Entre estos intentos de comprender la Fenomenología del espíritu en su relación con la filosofía crítica kantiana puede destacarse la obra de Robert Pippin, Hegel's Idealism. The Satisfactions of Self-Consciousness ${ }^{2}$. Pippin lee la Fenomenología vinculando la obra hegeliana con la tradición kantiana, tanto en lo respectivo a los problemas abordados como en lo que hace al método ${ }^{3}$. Pippin indica que la Fenomenología del espíritu debe ser leída en el marco de los problemas establecidos

\footnotetext{
${ }^{1}$ Cfr. WILLIAMS, Robert R., "Hegel and Transcendental Philosophy", en The Journal of Philosophy, Vol. 82, No. 11 (Nov., 1985), p. 595. Williams sostendrá que Hegel es un filósofo trascendental. Kenneth Westphal en Hegel's epistemological realism. A Study of the aim and method of Hegel's Phenomenology of spirit, Kluwer Academic Publishers, Dordrecht, 1989, p. 46 sostiene la utilización en la Fenomenología del Espíritu de argumentos trascendentales análogos a los kantianos y en el marco de una problemática común a la tradición crítica, mas con la diferencia fundamental de un presupuesto realista por parte de Hegel, según el cual, el filósofo estudiaría condiciones de posibilidad para la experiencia consciente que deben corresponder con la estructura efectiva del mundo real (autónomo al pensar).

${ }^{2}$ PIPPIN, Robert, Hegel's Idealism. The satisfactions of self-consciousness, New York, Cambridge University Press, 1989.

${ }^{3}$ En el presente trabajo, nos proponemos confrontar una lectura kantiana y anti-metafísica de la Fenomenología del espíritu. Por ese motivo, resulta necesario comparar aspectos centrales de la filosofía de Kant y Hegel. Esto no obsta a que en el recorrido de Kant a Hegel haya habido momentos de transición progresiva, más concretamente, autores que hayan hecho aportes que fueran posteriormente recogidos por sus sucesores y, en particular, por Hegel. En este sentido, no puede dejar de estimarse el valor de autores como Reinhold, quien ha pretendido fundamentar la filosofía crítica en una fenomenología de la conciencia. Es Reinhold, sostiene Hoyos, el primero en buscar una mejor deducción de las categorías, tarea que recogería el idealismo alemán posterior y, entre ellos, Hegel (cfr. HOYOS, Luis Eduardo, $E l$ escepticismo y la filosofía trascendental. Estudios sobre el pensamiento alemán a fines del siglo XVIII, Siglo del Hombre Editores, Bogotá, 2001, pp. 80-85). De igual manera, la Doctrina de la ciencia de Fichte - en sus distintas ediciones - puede ser leída como una reelaboración del yo trascendental y como una mejora de la deducción de las categorías. Colomer habla de una deducción "dialéctica" de las categorías kantianas en Fichte (cfr. COLOMER, Eusebi, El pensamiento alemán de Kant a Heidegger. Tomo II. El Idealismo: Fichte, Schelling y Hegel, Herder, Barcelona, 2006, p. 56). En ambos casos de reelaboración de tal deducción, se introduce en ella un carácter dinámico en las categorías en juego. Para atender a este recorrido de Kant a Hegel, puede consultarse las siguientes obras: HOYOS, Luis Eduardo, El escepticismo y la filosofía trascendental. Estudios sobre el pensamiento aleman a fines del siglo XVIII, Siglo del Hombre Editores, Bogotá, 2001. COLOMER, Eusebi, El pensamiento alemán de Kant a Heidegger, T. II El Idealismo: Fichte, Schelling y Hegel, Herder, Barcelona, 2006. BEISER, Frederick, The Fate of Reason. German Philosophy from Kant to Fichte, Hardvard University Press, Cambridge, 1993. DI GIOVANNI, George, HARRIS, H. S., Between Kant and Hegel. Texts in the Development of Post-Kantian Idealism, Hackett Publishing Company, Indianapolis/Cambridge, 2000. AMERIKS, Karl, Kant and the Fate of Autonomy. Problems in the Appropriation of the Critical Philosophy, Cambridge University Press, Cambridge, 2000.
} 
por el idealismo trascendental. En lugar de incurrir en el error de definir a Hegel como un autor post-kantiano con una metafísica pre-crítica -dice Pippin- debe llevarse a cabo una lectura que ponga a la Fenomenología en la línea de la tradición kantiana ${ }^{4}$. Así, el problema fundamental de la Fenomenología sería la vinculación entre ser y pensar. De esa manera, se caracteriza a la Fenomenología como una deducción trascendental que tendría como tarea establecer la legítima aplicación del pensamiento al $\operatorname{ser}^{5}$. En cuanto al método, la Fenomenología consistiría en una reiterada aplicación de argumentos trascendentales que, partiendo de un factum dado -la experiencia del objeto-, retrocede a sus condiciones de posibilidad, las que se van adicionando de forma sucesiva hasta alcanzar la totalidad de elementos que conforman y posibilitan nuestro conocimiento objetivo. Según esta interpretación, la sección "Autoconciencia" sería un análogo del yo trascendental kantiano, una condición de posibilidad más de la aplicación del pensamiento al ser ${ }^{6}$.

\footnotetext{
4 “Atribuirle a Hegel una moderada inteligencia filosófica debería hacerle dudar a uno antes de construirlo como un filósofo post-kantiano con una metafísica pre-crítica" Op. Cit. PIPPIN, R., Hegel's Idealism. The satisfactions of self-consciousness, p. 7. Por el contrario, Pippin sostendrá que "completar el proyecto kantiano por parte de Hegel implica mucha más continuidad, en especial en lo que respecta a la ruptura trascendental con la metafísica tradicional, de lo que se ha reconocido" Op. Cit. PIPPIN, R. Hegel's Idealism. The satisfactions of self-consciousness, p. 16.

${ }^{5}$ Op. Cit. PIPPIN, R. Hegel's Idealism. The satisfactions of self-consciousness, p. 38.

${ }^{6}$ Pippin no ha sido el único que se ha preocupado por la familiaridad y distanciamiento entre Kant y Hegel. Varios autores han advertido la importancia de detenerse en una consideración sobre el vínculo entre Kant y Hegel y, entiendo, muchos de ellos admiten que esta relación se caracteriza fundamentalmente por una apropiación y reelaboración hegeliana de elementos kantianos. En esta línea de lectura, podemos inscribir a Kreines, Longuenesse, Houlgate, de Boer, Hyppolite, entre muchos otros. En el caso de Kreines, el autor se propone defender una lectura metafísica de Hegel en franca oposición a la propuesta de Pippin. El autor coloca el foco de su investigación en un punto nodal de las filosofías de Kant y Hegel: la pretensión hegeliana de superar el límite epistemológico trazado por Kant, esto es, el conocimiento de lo en sí. En este sentido, el autor estudiará cómo la filosofía hegeliana pretende postular el conocimiento de lo en sí y, al mismo tiempo, rechazar a lo en sí por ser una mera abstracción, carente de fundamento. La articulación de estas dos intenciones se da a través de una crítica a la concepción kantiana del entendimiento humano como finito en oposición a un entendimiento divino intuitivo, cfr. KREINES, James, "Between the Bounds of Experience and Divine Intuition: Kant's Epistemic Limits and Hegel's Ambitions", Inquiry, vol. 50, No 3, junio, 2007, pp. 307-308, 314. En el caso de Hyppolite, hay una explícita referencia a una reapropiación hegeliana de la obra kantiana, particularmente de su lógica trascendental en la Ciencia de la lógica y del yo trascendental en la Fenomenología del espíritu, en lo que se da a llamar una extensión de la lógica trascendental, cfr. HYPPOLITE, Jean, Lógica y existencia, Herder, Barcelona, 1996, p. 78.

También en oposición a la propuesta de Pippin de considerar a la Fenomenología como una "deducción trascendental" y a la Ciencia de la lógica como una "deducción inmanente" que debería cumplir las funciones de una deducción metafísica (esto es, de exponer las categorías del pensar puro), Longuenesse propone entender a la Ciencia de la lógica como una deducción tanto trascendental como metafísica, esto es, la Lógica de Hegel tendría tanto la tarea de probar la aplicación del pensar al ser como la de exhibir el verdadero contenido semántico de los conceptos del pensar puro. Junto a esto, la autora encuentra en Hegel una reelaboración de importancia fundamental del yo trascendental kantiano. Tal reelaboración se
} 
A pesar de la necesidad de reconocer cierta vinculación de la Fenomenología con la filosofía trascendental kantiana, encontramos excesiva esta analogía. Una lectura kantiana de la Fenomenología del espíritu tiene deficiencias en tanto no puede explicar ni hacerse cargo de sus elementos más innovadores. En efecto, la propuesta de Pippin de insertar a la Fenomenología del espíritu en la tradición kantiana puede ser acertada en dos aspectos: en lo referente a sus objetivos y en el papel constitutivo de la objetividad por la conciencia.

Sin embargo, la descripción hegeliana de la conciencia en la "Introducción" a la Fenomenología se diferencia de la conciencia kantiana. Sus notas distintivas exigen llevar a cabo un método novedoso que no se encuentra en la obra kantiana ni es comprendido desde la concepción kantiana de conceptos tales como experiencia, objetividad, conocimiento y conciencia. Para comprender el proyecto fenomenológico pensado por Hegel debemos objetar las lecturas kantianas de la Fenomenología del espíritu e identificar con claridad sus objetivos, su método, su concepción de la conciencia, del objeto, del conocimiento y de la experiencia.

encuentra en la "doctrina del concepto", más precisamente, en la noción misma de concepto. Mientras el entendimiento kantiano se brinda a sí mismo un objeto del puro pensamiento de carácter meramente formal, el concepto hegeliano, caracterizado por abarcar lo distinto de sí, debe poder brindarse un objeto verdaderamente acorde a sí. En este punto, la autora considera que se trata aquí de una reelaboración del concepto de verdad y de una pretensión por parte de Hegel de superar el límite kantiano de la cosa en sí. Cfr. LONGUENESSE, Béatrice, Hegel's Critique of Metaphysics, Cambridge, Cambridge University Press, 2007, pp. 5, 20, 23, 27.

En el caso de Houlgate, el autor entiende la obra de Hegel como una lógica trascendental, esto es, como la explicitación de las condiciones de posibilidad del pensar constituyente de objetos, i.e. la estructura lógico-conceptual que conforma la estructura ontológica de los objetos de la experiencia, cfr. HOULGATE, Stephen, "Thought and Being in Kant and Hegel," The Owl of Minerva, 22, no. 2 1991, pp. 132-133, 140 and HOULGATE, Stephen, The opening of Hegel's Logic, Purdue University Press, Indiana, 2006, p. 436. Por su parte, de Boer, compartiendo en parte la lectura anterior, entiende que Hegel lleva a cabo una superposición de "analítica" y "dialéctica" en la que, de la primera, se rescata la intención kantiana de suplantar la vieja ontología por una nueva ontología de la experiencia (esto es, una "analítica trascendental") y, de la segunda, se rescata el carácter dialéctico de las categorías. Sin embargo, aunque en esto juegue un papel fundamental la apercepción, la autora niega la posición de Pippin consistente en entender a la filosofía hegeliana como un desarrollo del yo trascendental. Por el contrario, el yo trascendental es sólo un momento de un desarrollo dialéctico superior. Cfr. DE BOER, Karin, "The dissolving force of the concept: Hegel's ontological logic", The Review of Metaphysics, Vol. 57, No. 4, Jun., 2004, pp. 807, 812 y DE BOER, Karin, On Hegel. The sway of the negative, Palgrave Macmillan, Hampshire, 2010, p. 44.

Ahora bien, a pesar de estos tratamientos de las reelaboraciones conceptuales hegelianas de la obra de Kant, creo que en la bibliografía invocada no se ha advertido suficientemente el verdadero sentido del carácter dinámico introducido por Hegel en el pensar. Dicho en otras palabras, estas lecturas de la reelaboración hegeliana de puntos nodales de la filosofía de Kant no destacan, en mi opinión, el carácter dinámico de la experiencia y las consecuencias de ello al momento de leer la obra de Hegel en comparación con la de Kant. 
En concordancia con la interpretación mencionada entendemos que la Fenomenología pretende fundar la relación entre ser y pensar. Pero intenta lograrlo por fuera de los límites kantianos. Los primeros párrafos de la "Introducción" se ocupan de una crítica de la concepción kantiana del conocimiento, catalogada como "temor a la verdad"7. Las caracterizaciones del conocimiento como médium o como instrumento allí descritas presuponen "que lo absoluto se halla de un lado y el conocimiento de otro, como algo para sí y que, separado de lo absoluto, es, sin embargo, algo real" y que "el conocimiento, que, al ser fuera de lo absoluto es también [...] fuera de la verdad, es sin embargo verdadero" ${ }^{8}$. De este modo, nos encontramos con un conocimiento caracterizado como "separado de lo absoluto, y [...] un absoluto separado del conocimiento", cuando en verdad "solamente lo absoluto es verdadero o solamente lo verdadero es absoluto" ${ }^{\prime 9}$.

La caracterización kantiana del conocimiento objetivo como no correspondiendo a la cosa en sí obliga a relativizarlo, permitiendo ser catalogada como un escepticismo trascendental. El idealismo trascendental, al postular la posibilidad de una cosa en sí incognoscible distinta del objeto conocido, conduce al escepticismo trascendental, pues Kant no tiene

modo de establecer que las condiciones para una experiencia autoconsciente de objetos sean genuinamente objetivas. El resultado de sus respectivas deducciones o bien relativizaba sus afirmaciones sobre objetos a meros fenómenos o creaba una infinita e infinitamente inútil tarea, [...] una reconciliación que no puede ocurrir nunca [entre ser y pensar $]^{10}$.

La postulación de la cosa en sí implica distinguir conocimiento objetivo y verdad (absoluto). La propuesta kantiana es vista por Hegel como caracterizando al conocimiento como meramente relativo y limitado a meros fenómenos, mientras a la vez se supone un verdadero absoluto incognoscible ${ }^{11}$. La vinculación entre ser y pensar

\footnotetext{
7 HEGEL, G.W.F., Fenomenología del espíritu, Madrid, ABADA / UAM Editores, ed. bilingüe de Antonio Gómez Ramos, 2010, p. 145.

${ }^{8}$ Loc. cit.

${ }^{9}$ Loc. cit.

${ }^{10}$ Op. Cit. PIPPIN, R., Hegel's Idealism. The satisfactions of self-consciousness, p. 92.

11 "Hegel reprochaba a Kant haber fragmentado la verdad en un sistema dualista de oposiciones intelectuales abstractas sin vía de conciliación (sujeto-objeto, intelecto-razón, materia-forma, cosa en sí-
} 
propuesta por la Fenomenología tiene la pretensión de superar esta distancia insalvable ideada por el kantismo.

Hegel deberá enfrentarse al escepticismo trascendental superando esta distinción entre conocimiento y absoluto que constituía una separación infranqueable entre ser y pensar. La tarea de la Fenomenología será demostrar sucesivamente que los distintos aspectos del objeto que se presenten como independientes al pensar son, en efecto, productos del pensar y no autónomos a la conciencia. "La conciencia alcanzará un punto en el que se desprenda de su apariencia de arrastrar consigo algo extraño que sólo es para ella y lo es en cuanto otro" ${ }^{12}$. Con esto, se alcanzaría al final del recorrido fenomenológico: la identificación absoluta entre ser y pensar.

Así, el proyecto hegeliano se encuentra comprometido con alcanzar lo absoluto mismo y su identificación con el saber: lo absoluto al que debe conducirse Hegel no puede excluir al conocimiento, porque "sólo lo absoluto [es] verdadero, o [...] sólo lo verdadero [es] absoluto"13. De este modo, Hegel señala el recorrido de la Fenomenología como "el camino de la conciencia natural acuciada por llegar al saber verdadero" ${ }^{14}$. Este verdadero saber, en tanto que sólo es verdadero lo absoluto, es lo absoluto mismo. Alcanzar el verdadero saber significaría alcanzar un saber que no se encuentra escindido de lo absoluto y, por tanto, que no halla un mejor conocimiento ni una verdad fuera de sí. El verdadero saber como meta de la Fenomenología (1) "está allí donde el saber ya no tenga necesidad de ir más allá de sí mismo", (2) "donde se encuentre a sí mismo, y" (3) donde "el concepto corresponda al objeto, y el objeto al concepto" $^{\prime 15}$. Esta caracterización de la meta de la Fenomenología es a su vez una caracterización del verdadero saber o de lo absoluto.

La primera nota es una descripción del carácter absoluto del saber e implica la completitud del mismo, esto es, que no hay objeto que no se encuentre en el saber

pensamiento)". FABRO, Cornelio, La Dialéctica de Hegel, Editorial Columba, Bs. As., 1969, p. 58. En concordancia con esta posición, cfr. HEGEL, Ciencia de la lógica, Ed. Solar, Bs. As, Trs. A. y R. Mondolfo, 1976, pp. 47 y 57.

${ }^{12}$ HEGEL, Fenomenología del espíritu, p. 161.

${ }^{13}$ Op. cit. p. 145.

${ }^{14}$ Op. cit. p. 147.

${ }^{15}$ Op. cit. p. 151. 
mismo, no hay objeto por fuera o extraño al saber. Es un saber que no tiene un más allá de sí, por fuera del cual haya algo, abarca todo cuanto es.

La segunda caracterización señala el aspecto reflexivo del saber. El saber por fuera del cual no hay nada debe abarcarse a sí mismo a fin de ser un saber de sí mismo. De lo contrario, sería un saber al que le faltaría la concepción de su propio saber y, por tanto, no absoluto. Esto nos indica que, por un lado, la Fenomenología como investigación de la vinculación entre ser y pensar no sólo estudiará la caracterización del objeto (ser), sino también se ocupará del saber del saber, de la caracterización del propio saber. Por el otro, adelantamos que la "Autoconciencia" aporta el carácter reflexivo que esta caracterización del saber supone. En tanto que la autoconciencia es un saber de sí de la conciencia deberá brindar algún elemento relevante para la caracterización del saber como reflexivo.

La tercera caracterización retoma las anteriores a la vez que permite volver a formular los objetivos de la Fenomenología en el lenguaje de la tradición idealista. La meta buscada es la identidad entre concepto y objeto o entre ser y pensar. Pero dicha identificación implicará descubrir que no hay objeto fuera del saber y que el verdadero objeto del saber no es algo diferente al saber mismo: el objeto conocido por el saber es el saber mismo, la estructura conceptual que constituye la estructura ontológica del objeto. Esta meta se alcanza cuando la estructura ontológica sea tal que no permita la conformación de un objeto que se presente como independiente al saber que lo constituye. Este reconocimiento de la identidad y concordancia de estructura conceptual de la conciencia y estructura ontológica del objeto es una identificación de objeto y saber, pero también un conocimiento del saber por el saber mismo que no permite la postulación de nada externo a él.

Como puede verse, el objetivo de vincular ser y pensar toma en Hegel un sentido más amplio que en la propuesta filosófica kantiana. Debemos considerar la caracterización hegeliana de la conciencia, pues en el tratamiento de la conciencia y su objeto de conocimiento es donde encontraremos una explicación de cómo "saber" y "objeto" se relacionan. 
Atendiendo a las descripciones que Hegel ofrece de la conciencia puede comprenderse las sospechas de los comentaristas de hallar aspectos kantianos en la Fenomenología. En la Fenomenología del espíritu la conciencia es constitutiva de la objetividad, siguiendo así a la filosofía crítica ${ }^{16}$. La estructura ontológica del objeto es dependiente de la estructura conceptual de la conciencia. Sin embargo, Hegel introduce un aspecto dinámico en la estructura de la conciencia que modifica el modo de concebir el objeto, la experiencia, el sujeto y el conocimiento, alejándose así de la filosofía crítica, incluso en el método filosófico.

La estructura conceptual de la conciencia a cuyo cargo está la constitución de la objetividad es, en la filosofía kantiana, de naturaleza estática. La Crítica de la razón pura no concibe un objeto que sufre modificaciones en su estructura ontológica ni una conciencia cuya estructura categorial sufre alteraciones. El propio tipo de argumentación con el que se desarrolla la "Deducción Trascendental", pieza fundamental de la Crítica en la demostración de la aplicación del pensar al ser, supone el carácter estático del objeto de conocimiento. En la deducción Kant se vale de un argumento trascendental consistente en probar que las categorías son condiciones de posibilidad de la experiencia. Así, la experiencia permanece como un factum, como lo dado, estático e imperturbable. Frente a ello, sus condiciones de posibilidad se van estipulando y agregando, como elementos estáticos y fijos, que no pueden volver a ser alterados. Como veremos, la conciencia hegeliana incluirá un carácter dinámico que exige abandonar este método filosófico.

Hegel describe una conciencia que "distingue de sí algo a lo que, a la vez, se refiere $^{\prime 17}$. En esta estructura de la conciencia podemos diferenciar un saber del objeto y el objeto mismo referido por el saber, lo que Hegel llama "para sí" y "en sí" o verdad. Mientras lo en sí es el presunto objeto inmediato de la conciencia, el para sí es el

\footnotetext{
${ }^{16}$ Según Fabro, Hegel aprobaría "el descubrimiento de Kant [consistente] en haber puesto el Yo en el principio originario de la objetividad", Op. Cit. Fabro, La Dialéctica de Hegel, pp. 50 y ss. De igual modo, Hyppolite señala al principio aperceptivo kantiano como el punto de partida del idealismo alemán posterior, al mismo tiempo en que repara en que "la dialéctica trascendental, al condenar toda metafísica del objeto, del ser (...) contenía en germen una metafísica de otro tipo, una metafísica del sujeto", superando de esta manera la limitada opinión de que sólo el principio aperceptivo constituye la herencia kantiana de la filosofía hegeliana. HYPPOLITE, J., Génesis y estructura de la Fenomenología del espíritu, Ediciones 62, Barcelona, 1991, pp. 131 y 132.

${ }^{17}$ HEGEL, Fenomenología del espíritu, p. 153.
} 
carácter reflexivo del saber, la referencia del objeto conocido a su conocimiento, como su fundamento. En esta estructura en que diferenciamos un polo subjetivo y otro objetivo, podemos reconocer que "ser para otro y ser en sí mismo, caen ambos dentro del saber que estamos investigando" ${ }^{18}$, i.e. saber y objeto son momentos de la propia conciencia que sabe y constituye al objeto de su saber.

Pero la constitución del objeto por parte del saber no equipara a Hegel con la filosofía kantiana. Hegel nos indica que "la distinción que se acaba de hacer [entre concepto y objeto, o en sí y para sí] tiene lugar dentro de ella [la conciencia]"19, es decir, la distinción y comparación que se haga entre saber y verdad (objeto) son momentos de la conciencia. En consecuencia, "la naturaleza del objeto que estamos investigando dispensa de [überhebt, rebasa] esta separación o de esta apariencia de separación" y "la conciencia aplica en ella misma su patrón de medida, con lo que la investigación será una comparación de ella consigo misma" ${ }^{20}$.

Aquí Hegel expresa, quizá, el punto fundamental que nos permite comprender cómo se introduce un carácter dinámico en el análisis de la relación entre objeto y conciencia. En tanto objeto y saber son polos de una misma conciencia, la pauta de la adecuación del saber al objeto la da la propia conciencia en una comparación consigo misma, sin acudir a un factor estático externo. Si en tal examen del saber, objeto y saber no se correspondieran, la conciencia deberá alterar su saber para adecuarlo a su objeto. No obstante, Hegel advierte que

al alterar el saber [para adecuarlo al objeto], se le altera a la conciencia también el objeto mismo; pues el saber que hay es, esencialmente, un saber acerca del objeto; con el saber, también el objeto deviene otro, pues él pertenecía esencialmente al saber. Y así le resulta a la conciencia que aquello que antes era lo en sí, no es lo en sí, o que sólo era en sí para ella ${ }^{21}$.

El objeto no puede evadir su naturaleza de objeto constituido por el saber de la conciencia $y$, consecuentemente, el fracaso del saber que lo constituía implica una alteración del objeto, en su ontología, en lo que era ser objeto. El examen del saber es

\footnotetext{
${ }^{18}$ Op. cit. 155.

${ }^{19}$ Loc. cit.

${ }^{20}$ Loc. cit.

${ }^{21}$ HEGEL, Fenomenología del espíritu, p. 157.
} 
a la vez un examen de la pauta del saber (el objeto) y la alteración de la estructura conceptual de la conciencia es la modificación de la estructura ontológica del objeto. En palabras de Hegel,

al encontrar, entonces la conciencia en su objeto que su saber no corresponde a éste, el objeto mismo tampoco se sostiene; o bien, el patrón de medida del examen se altera si aquello de lo cual él debía ser patrón no resiste el examen; y el examen no sólo es un examen del saber, sino también de su patrón de medida ${ }^{22}$.

Hegel describe un paso más que da la conciencia al descubrir y diferenciar el en sí (objeto) del en sí para sí (objeto del saber). "La conciencia sabe algo; este objeto es la esencia o lo en sí, pero también es lo en sí para la conciencia; y así hace entrada la ambigüedad de esto verdadero" ${ }^{23}$. Dicha ambigüedad consiste en que la conciencia tiene "dos objetos: uno, el primer en sí, y luego, el ser para ella de este en sí" 24 . "A la conciencia se le altera el primer objeto; éste deja de ser lo en sí, y deviene a sus ojos un objeto tal que sólo para ella es lo en sí" y esta conversión o anulación del primer objeto "es la experiencia hecha sobre él" ${ }^{25}$. Con el descubrimiento del carácter para sí de lo que antes se postulaba como un objeto autónomo, se da el emerger de un nuevo objeto, que es vivido por la conciencia como si contingentemente surgiera ante ella otro objeto. Este nuevo objeto en verdad ha llegado a ser por una inversión de la conciencia, es decir, por una experiencia sufrida por la conciencia en la cual ella, su saber y su objeto han sufrido la conversión descrita. Esta experiencia es un "movimiento dialéctico que la conciencia ejecer en ella misma, tanto en su saber como en su objeto, en la medida en que, a partir de él, le surge a ella el nuevo objeto verdadero" $^{\prime 26}$.

Cada nueva figura de la conciencia o estructura conceptual, impulsada por el descubrimiento de que aquello que se consideraba en sí no era otra cosa que para sí, implica una nueva caracterización y constitución de la ontología del objeto. Cada cambio del saber (cada figura de conciencia) posee una nueva esencia, una nueva

\footnotetext{
${ }^{22}$ Loc. cit.

${ }^{23}$ Loc. cit.

${ }^{24}$ Loc. cit.

${ }^{25}$ Op. cit. 159.

${ }^{26}$ HEGEL, Fenomenología del espíritu, p. 157.
} 
verdad, una nueva concepción de lo que es, y por tanto un nuevo tipo de objeto ${ }^{27}$. La serie de las conversiones que sufre la conciencia se encuentra impulsada por una necesidad fundada en la meta a alcanzar. El saber absoluto, la identificación plena de concepto y objeto, implica la imposibilidad de caracterizar al objeto del saber de la conciencia como un en sí autónomo. La experiencia dialéctica antes descrita tiene a cargo alcanzar una constitución del objeto tal que implique su plena identificación con el saber, impidiendo dejar restos independientes o autónomos al pensar. El último movimiento de la experiencia de la conciencia considerado consistía en el emerger de un aparente nuevo objeto. Esto implicaba que la tarea de la conciencia no se había cumplido plenamente: la conciencia descubría que el objeto era de su saber, pero ante esta nuevo saber y nueva configuración de la objetividad surgía un nuevo objeto en sí. La identificación plena entre concepto y objeto exige el descubrimiento por parte de la conciencia de que todo en sí no es más que para sí, que toda caracterización de lo que es se identifica absolutamente con el pensar.

A partir de este estudio de la dinámica interna a la conciencia hegeliana puede advertirse la inadecuación de una lectura kantiana de la Fenomenología. En primer lugar, la Fenomenología no supone una caracterización de la experiencia como un dato estático sobre el que se puedan impulsar argumentos trascendentales, que ofrezcan condiciones de posibilidad de tal experiencia. Hegel innova al concebir una experiencia dinámica y dialéctica, junto a una conciencia que se altera en su estructura conceptual en conjunción con la efectiva modificación de la estructura ontológica de su objeto. Retroceder desde un factum hacia las condiciones de posibilidad no es viable para una experiencia dialéctica en la que la estructura ontológica del objeto cambia. Tal metodología tampoco podría suponer una superación del escepticismo trascendental, pues se limitaría a descubrir condiciones subjetivas del pensar que no garantizarían

\footnotetext{
27 "[E]n cada estadio de la Fenomenología se constituye una cierta noción de la objetividad, una verdad propia de ese estadio. No se trata tanto de pensar objetos individuales como de determinar el carácter de una cierta forma de objetividad" HYPPOLITE, Génesis y estructura de la Fenomenología del espíritu, Ediciones 62, Barcelona, 1991, p.146.

Cfr. HEGEL, Fenomenología del espíritu, p. 157 "En tanto que lo que primer aparecía como el objeto se degrada, a los ojos de la conciencia, a un saber acerca de él, y en tanto que lo en sí se convierte en un ser de lo en sí para la conciencia, este ser es el nuevo objeto, con el cual también entra en escena una nueva figura de la conciencia, para la cual la esencia es algo distinto de lo que lo era para la figura precedente. Es esta circunstancia la que guía toda la serie de figuras de la conciencia en su necesidad".
} 
que reste algo incognoscible existente en sí. La concepción de una experiencia dialéctica en la que la estructura ontológica del objeto y conceptual de la concienca se ven alteradas en función de impedir toda postulación de un objeto autónomo e incognoscible garantiza la consecución de los objetivos fenomenológicos y toma distancia de la filosofía crítica, tanto en cuanto a su meta como en cuanto al método.

Ahora bien, la meta final de la Fenomenología se encuentra en la identificación absoluta de ser y pensar. Tal identificación sólo puede alcanzarse si puede atribuirse el pensar mismo al ser. El capítulo dedicado a la "autoconciencia" se ocupa precisamente de esto.

\section{La "Autoconciencia" y la primera constitución del ser como pensar}

La "Autoconciencia" introduce una serie de cuestiones de naturaleza práctica innegable ${ }^{28}$. Sin embargo, en el marco de esta investigación nos interesa analizar qué aporte ofrece este capítulo a los objetivos generales diagramados en la "Introducción" y estipulados en la sección anterior. Esto supone continuar nuestro enfoque teorético, haciendo especial hincapié en los aspectos gnoseológicos y metafísicos de esta sección.

Los primeros párrafos de la "Autoconciencia" indican que nos encontramos ante una nueva figura que presentará un cambio radical en el modo de concebir el objeto. Devenir autoconciencia significa poseer un saber de sí misma tomándose por objeto. Sabiéndose conciencia, ésta descubre que el objeto, caracterizado como en sí en la sección anterior, se revela como mero para otro, esto es, objeto del saber. Mientras en la sección "Conciencia" "lo verdadero [el objeto] le es a la conciencia algo otro de ella misma", aquí "el objeto [...] resulta ser un modo en el que él, el objeto, es para otro" 29 . El descubrimiento de que el objeto es objeto del saber de la conciencia y no un en sí autónomo a ella es un conocimiento sobre la propia naturaleza de la

\footnotetext{
${ }^{28}$ La intromisión de conceptos tales como 'vida', 'apetito' (o 'deseo'), 'muerte' y 'reconocimiento' hacen innegable su aspecto práctico. Axel Honneth, La lucha por el reconocimiento. Por una gramática moral de los conflictos sociales, Ed. Crítica Grijalbo Mondadori, Barcelona, 1997 y Alexandre Kojéve, La dialéctica del amo y del esclavo en Hegel, Fausto, Buenos Aires, 1996 ofrecen lecturas en este sentido. También sigue lineamientos similares PIPPIN, Hegel on Self-consciousness. Desire and Death in the Phenomenology of Spirit, Princeton University Press, New Jersey, 2011.

${ }^{29}$ Op. Cit. HEGEL, Fenomenología del espíritu, p. 245.
} 
conciencia, es un saber sobre el saber mismo, con lo cual "la certeza se es a sí misma su propio objeto" ${ }^{30}$, es decir, el propio saber se toma como objeto de conocimiento. Estamos frente a un saber del saber que implicará una modificación (1) en la concepción del objeto conocido, (2) en la conciencia que sabe y (3) en la concepción del conocimiento.

El objeto sufre alteraciones en tanto no puede seguir conservándose como mero en sí; la conciencia, por su parte, no puede mantener su relación inmediata con el objeto, pues éste se le presentará como mera negatividad-como veremos más adelante-; por último, el saber será "de sí mismo" a diferencia de la sección anterior ("el saber de otro", del objeto) ${ }^{31}$.

Esta serie de conversiones que a continuación profundizaremos están en plena concordancia con la "Introducción" de la Fenomenología. En tanto la conciencia se dirija a una nueva figura modificando su saber, sufrirá el emerger de un nuevo objeto en concordancia con tal saber. Alcanzar un saber según el cual el objeto deja de caracterizarse como en sí para ser considerado para sí implica convertirse en autoconciencia, pues supone alcanzar un saber sobre un objeto sapiente, sobre lo que es ser conciencia, y es descubrir que el objeto es para su saber, es para la conciencia. Es apropiado llamar a este saber "autoconciencia", pues es el saber sobre el saber del objeto, y por tanto, no se orienta al objeto del saber, sino a la propia conciencia, al saber $^{32}$.

Así, Hegel afirma que "la certeza se es a sí misma su propio objeto, y la conciencia se es a sí misma lo verdadero". La conciencia se ha convertido en saber del saber, y por tanto, el saber (certeza) es su objeto y ella misma como tal saber se presenta ante sí misma como objeto (lo verdadero). Pero esta afirmación posee un segundo sentido más general que permite comprender el aporte que la "Autoconciencia" hará al recorrido fenomenológico. La conversión de la conciencia en "lo verdadero", que la certeza sea "su objeto", anticipa la experiencia fenomenológica que la conciencia sufrirá en este capítulo. Si leemos esta afirmación como una

\footnotetext{
${ }^{30}$ Loc. cit.

${ }^{31}$ Loc. cit.

${ }^{32}$ Cfr. LABARRIERE, Pierre-Jean, La Fenomenología del Espíritu de Hegel. Introducción a una lectura, Fondo de Cultura Económica, México, 1979, p. 100.
} 
indicación general de la tarea a emprenderse en la "Autoconciencia", implica que debe emerger propiamente en la experiencia el saber mismo como objeto, como aquello que es, como siendo algo.

Las figuras anteriores de la conciencia permitían sólo la experiencia de meros objetos. Encontrábamos saberes que definían al objeto como en sí y la conciencia experimentaba objetos carentes de saber y reflexión. El saber de la nueva figura de la conciencia permite la emergencia de un nuevo tipo de objeto, capaz de saber y de reflexión. Con el emerger de tal nuevo objeto como poseedor de saber, encontraríamos una primera vinculación fundamental entre ser y saber; estaríamos ante el primer surgir del saber en el ser mismo. Nos encontraríamos por primera vez con una caracterización del ser que lo vuelve saber.

En esta nueva figura que Hegel califica como "saber de sí mismo" el saber de otro se ha perdido, ha desaparecido, aunque "sus momentos se han preservado" ${ }^{33}$. Las formas del ser del objeto "no son ya como esencias, sino como momentos de la autoconciencia" ${ }^{34}$. Los momentos o formas del objeto inmediato de la conciencia se evaporan en tanto formas de aquello diferente de la conciencia, dejan de ser momentos del objeto para pasar a ser momentos de la propia conciencia y dejan, por tanto, de ser diferentes de la conciencia misma. Esto es afirmar que el objeto de la conciencia deja de subsistir como diferente e independiente a la conciencia.

Sin embargo, la autoconciencia es un movimiento de reflexión desde lo otro de sí hacia sí, y por tanto requiere de tal otro. La autoconciencia debe conservar de alguna forma el objeto inmediato. Así, la autoconciencia tendrá "un objeto doble: uno, el inmediato [...], y el segundo, [...] sí misma" ${ }^{35}$. Por un lado, este objeto inmediato no puede conservarse como mero en sí autónomo al saber. Por el otro, este primer objeto no puede desvanecerse completamente. Sin él, la autoconciencia no es autoconciencia. Por eso, "para ella [la autoconciencia], hay el ser-otro en cuanto un ser, o en cuanto momento diferenciado", y con la conservación de este ser-otro "la autoconciencia queda conservada como conciencia, y se conserva para ella toda la

\footnotetext{
${ }^{33}$ Op. Cit. HEGEL, Fenomenología del espíritu, p. 245.

${ }^{34}$ Op. Cit. HEGEL, Fenomenología del espíritu, p. 247.

${ }^{35}$ Loc. cit.
} 
extensión del mundo sensible" ${ }^{36}$. En otras palabras, la autoconciencia supera pero también conserva la relación inmediata de la conciencia con su objeto.

Así, la autoconciencia se diferencia respecto del ser otro (objeto). Por medio de esta diferenciación, la autoconciencia retorna hacia sí desde lo diferente. Mas, este primer momento debe estar referido a un segundo momento, en el que se establece "la unidad de la autoconciencia consigo misma", esto es, "la unidad de ella misma con esta diferencia" ${ }^{37}$, su reconocimiento de esto otro como idéntico a sí misma. En otras palabras, la autoconciencia necesita de lo extraño para conformar su identidad; pero necesita, también, referir aquello extraño a sí misma. Así, encontramos dos momentos de la conciencia (su diferenciarse y su identidad consigo) y dos objetos (el objeto inmediato y sí misma).

En esta estructura de dos momentos de la autoconciencia y dos objetos la autoconciencia es caracterizada como 'deseo' o 'apetito' [Begierde]. El objeto inmediato pierde su carácter esencial o verdadero y queda absorbido como mero momento del saber. Esta forma de la conciencia según la cual se niega el ser del objeto es llamada deseo. Pero esta forma de la conciencia se encuentra con que su verdadera esencia (la cual ya no es el objeto inmediato, sino el segundo objeto, esto es, sí misma) "sólo está presente [...] en la oposición del primer objeto" ${ }^{38}$. En otras palabras, el objeto inmediato de la autoconciencia es necesario para su conformación como saber de sí; pero, al mismo tiempo, es inesencial. No es el objeto acorde a su nuevo saber, no es la realización del nuevo saber de la conciencia (un saber de sí). El objeto efectivo (esencia) de la autoconciencia debería ser una autoconciencia.

Sin embargo, su saber de sí no se presenta como objeto. El objeto es aun únicamente el objeto negado (inmediato) y la autoconciencia sólo se manifiesta a sí misma como mera oposición a éste, sin tener realidad en el ser. En tanto esta forma de la conciencia es mera negación de su objeto inmediato, no puede tener por objeto a su verdadera esencia (un objeto autoconsciente), concordante con su saber autoconsciente. Así, la sección "Autoconciencia" se presentará "como el movimiento

\footnotetext{
${ }^{36}$ Loc. cit.

${ }^{37}$ Loc. cit.

${ }^{38}$ Loc. cit.
} 
en el que esta oposición [frente al primer objeto] quede cancelada y asumida [aufgehoben], convirtiéndosele en la igualdad de sí misma consigo misma” ${ }^{39}$, es decir, como el proceso por medio del cual aquello opuesto a la autoconciencia debe llegar a presentarse como idéntico a ella. Esto no se logrará en tanto no se consiga la caracterización del objeto como autoconciencia.

La realización de la identidad de la autoconciencia y su objeto comienza con un movimiento interno o reflexión en el propio polo objetivo. Así como la conciencia retorna desde lo otro de sí hacía sí misma, el objeto emula esa reflexión y se presenta como vida. "[E]I objeto, que es lo negativo para la autoconciencia [...] ha retornado hacia dentro de sí, en la misma medida en que la conciencia también lo ha hecho por la suya. A través de esta reflexión hacia dentro de sí, el objeto ha llegado a ser vida"40. Así como la autoconciencia se concibe como autónoma a su objeto, el objeto reflexionado será concebido por la propia conciencia como un ser autónomo.

[T]anto [...] como se sostiene en sí misma y es autónoma la conciencia, se sostiene en sí mismo y es autónomo, en sí, su objeto. Por eso, la autoconciencia, que es para sí sin más, y marca a su objeto inmediatamente con el carácter de lo negativo, [...] hará [...] la experiencia de la autonomía del objeto ${ }^{41}$.

Con el tratamiento de la vida, damos un primer paso hacia la conversión del en sí negado en reflexión. La autoconciencia que se oponía a su objeto y era mera certeza de sí encuentra un objeto acorde a su saber en la figura de la vida, postulando algo, allí en el ser, tan autónomo como ella.

La autoconciencia sufrirá un movimiento especular en su objeto. Como determinado en concordancia con el saber, el objeto emula los movimientos de la conciencia y se vuelve un en sí reflexionado: "es ser reflexionado dentro de sí, y el objeto del deseo inmediato es algo vivo" ${ }^{42}$. La unidad conformada por la autoconciencia y lo negado se escinde en autoconciencia y vida (ser en sí reflexionado y autónomo) $)^{43}$.

\footnotetext{
${ }^{39}$ Loc. cit.

${ }^{40}$ Op. Cit. HEGEL, Fenomenología del espíritu, p. 247.

${ }^{41}$ Op. Cit. HEGEL, Fenomenología del espíritu, p. 249.

${ }^{42}$ Op. Cit. HEGEL, Fenomenología del espíritu, p. 247.

${ }^{43}$ Op. Cit. HEGEL, Fenomenología del espíritu, p. 249.
} 
En el desarrollo de este nuevo objeto (objeto vivo) alcanzaremos la realización efectiva de la verdadera esencia de la autoconciencia. En otras palabras, alcanzaremos la presentación en el polo objetivo de otra autoconciencia. Comencemos por su primer momento de determinación, consistente en

la infinitud en cuanto el estar canceladas [Aufgehobensein] todas las diferencias, el movimiento puro alrededor de un eje, la quietud de sí misma en cuanto infinitud absolutamente inquieta; la propia autonomía de sostenerse por sí misma, donde están disueltas las diferencias del movimiento ${ }^{44}$.

Este primer momento consiste en la mera autonomía indiferenciada de lo vivo, que entra en juego junto al segundo momento de "las diferencias [que] están en este medio simple en cuanto diferencias" ${ }^{45}$, es decir, las diferentes figuras vivas que especifican aquella primera figura universal e indeterminada. La vida se presenta como la relación de estos dos momentos: (1) "esta fluidez, en cuanto autonomía igual a sí misma, [que] es ella misma el subsistir, o la substancia de" (2) "esos diferentes, donde ellos, por tanto, están como miembros diferenciados y partes que son para si" ${ }^{46}$.

"Los miembros autónomos" o figuras autónomas que encuentran subsistencia en la fluidez universal poseen una estructura reflexiva y brindan a ésta -en la que subsisten- tal carácter reflexivo. Dicha reflexividad es una emulación, en el polo objetivo, del carácter negativo de la autoconciencia. Así como la autoconciencia niega su objeto para constituir su identidad, las figuras autónomas de la vida deben negar la indiferenciación de la mera fluidez universal de la vida ${ }^{47}$. Por su parte, la sustancia de la fluidez universal se divide en figuras y les brinda su autonomía. La sustancia obtiene la reflexividad gracias a las figuras autónomas y éstas obtienen de aquélla subsistencia y fluidez, esto es, la posibilidad de perdurar a través del gestarse y perecer. Recordemos que Hegel hablaba de "unidad" cuando caracterizaba el vínculo de la autoconciencia con su polo objetivo puramente negativo. Aquí nos encontramos

\footnotetext{
${ }^{44}$ Loc. cit.

${ }^{45}$ Loc. cit.

${ }^{46}$ Loc. cit.

47 Op. Cit. HEGEL, Fenomenología del espíritu, p. 251. La figura autónoma "niega esta fluidez y continuidad con ella [la substancia universal] y afirma de sí que no está disuelta en esto universal, sino que, más bien, se mantiene por particularizarse y separarse de esta naturaleza inorgánica suya, y consumirla".
} 
nuevamente con la unidad, referida esta vez al polo objetivo mismo. Tal como la autoconciencia se presentaba como unidad entre su certeza y su esencia, aquí, el polo objetivo se presentará como unidad de dos elementos que le son propios y emulará movimientos análogos a los de la autoconciencia. La vida como universalidad es sustancia; pero también negatividad, en tanto las figuras singulares (reflexivas) pueden emerger en tanto se diferencian de esta universalidad. Esta universalidad les da subsistencia, pero también fluidez: permite el surgir, perdurar y perecer sin que lo subsistente se pierda. Por su parte, la fluidez universal sólo puede manifestarse en estas formas particulares, pues como mera fluidez universal no es nada.

Si bien la fluidez o vida universal es en principio lo que es negado y superado por la figura particular (así como el objeto es para la autoconciencia), la vida "Ilega [...] a ser lo otro a través de esta diferencia" ${ }^{48}$. La vida universal no se elimina por la negación impulsada por la figura particular. Por el contrario, la vida universal vive en la figura diferenciada. Así como la autoconciencia llegará a ser en lo otro (en el polo objetivo), vemos ahora un movimiento anticipatorio en el polo objetivo. La vida universal es en y a través de cada figura diferenciada.

Esta identificación da por resultado que "la substancia simple de la vida [...] [sea] la escisión de ella misma en figuras, y a la vez, la disolución de estas diferencias subsistentes; y la disolución de la escisión es [...] un escindir, o un articular en miembros diversos [ein Gliedern] ${ }^{\prime 49}$. Con su conversión en un momento de la vida universal, la figura particular se convierte en una figura a la vez universal. Se vuelve género de vida que adquiere un carácter universal. En esto consiste, precisamente, la subsistencia que ella adquiere de la vida universal.

La vida, combinando su sustancialidad indiferenciada y sus figuras autónomas, es "todo este recorrido cíclico [...], el conjunto de todo que se desarrolla, disuelve su desarrollo y se mantiene simplemente en este movimiento" ${ }^{50}$. La unidad de figura autónoma y fluidez universal permite que la figura sea puesta en movimiento, en identidad consigo misma a través de los cambios. La figura autónoma que se limitaba a

\footnotetext{
${ }^{48}$ Loc. cit.

${ }^{49}$ Loc. cit.

${ }^{50}$ Op. Cit. HEGEL, Fenomenología del espíritu, p. 253.
} 
diferenciarse de la sustancia universal, una vez identificada con ésta puede conservar su identidad a través del cambio y del fluir, así como la vida general conserva su identidad más allá de toda particularidad. Así, la sustancialidad de la vida que se manifiesta sólo en figuras particulares supone la identidad a partir del cambio, de la diversidad, del surgir de los individuos y de su perecer. Lo mismo sucede en cada figura particular, convirtiéndose ésta en un género. Esto significa poder reconocer a través del generarse y perecer no ya la identidad de un individuo, sino de una especie o género.

Así, encontramos en el polo objetivo una estructura reflexiva que comienza como un mero acto de superación o negación; pero que, volviendo sobre sí y unificándose con lo negado, produce una identidad que no se agota en el individuo, sino que puede apropiarse de aquello que se presentaba como extraño. Dicho de manera menos abstracta, las figuras particulares y autónomas de la vida que procuraban negar la mera vida indiferenciada, logran unificarse con ella, dando por resultado no ya la identidad de un único individuo que perecerá, sino reconociendo su identidad a lo largo de todo lo general y cambiante.

Con la reunión de los dos momentos de la vida, se vuelve a una unidad que "remite hacia algo distinto de lo que ella es, a saber, a la conciencia para la cual es como esta unidad, o como género" ${ }^{\prime 51}$. Hegel sólo ha descrito el movimiento del polo objetivo. Esta secuencia de movimientos termina con el emerger del objeto como género vivo y su referencia a la autoconciencia, la que Hegel no duda en llamar también "vida". Así, la vida remite nuevamente hacia algo otro de sí, para lo cual ella se presenta como género: este proceso de determinación objetiva concluye retornando a la autoconciencia.

Por su parte, la conciencia se beneficia de este desarrollo dialéctico llevado a cabo sobre su polo objetivo. Sin el emerger del género la conciencia no podría ser objeto para otra autoconciencia, pues resultaría imposible que la autoconciencia reconozca aquello extraño como un yo. Si el yo no fuera un género de vida y sólo

\footnotetext{
${ }^{51}$ Loc. cit.
} 
tuviéramos configuraciones objetivas individuales, la autoconciencia sólo podría catalogarse a sí como yo, y nunca podría reconocer a un otro como un "yo".

Así como el objeto sufrió conversiones análogas a los movimientos del saber de la conciencia, la autoconciencia sufre alteraciones concordantes con aquellas padecidas por su objeto. En primer lugar, Hegel llama "vida" a la autoconciencia.

Esta otra vida para la que es el género como tal, y que es ella género para sí misma, la autoconciencia, sólo se es a sí, primeramente, como esta esencia simple, y, en cuanto puro yo, se tiene a sí por objeto ${ }^{52}$.

Tal como el objeto inmediato de la autoconciencia se volvió vida y género, la autoconciencia modifica su saber de sí, altera su estructura conceptual de forma tal de comprenderse como vida y género. Con esto la autoconciencia pasa a ser algo vivo; no sólo se le presentan objetos vivos, ella misma se convierte en vida.

Sin embargo, este avance es mucho más lento de lo que parece. Si bien "la autoconciencia [...] en cuanto puro yo, se tiene a sí por objeto" ${ }^{53}$, no tiene aun conciencia de otros objetos autoconscientes ni se tiene a sí como objeto efectivo (esencia). Aun falta otro paso. Hegel analizó el movimiento de la vida, el proceso de determinación propio del polo objetivo. Pero la autoconciencia no modifica inmediatamente la naturaleza de su objeto, no al menos sin antes modificar su saber, y como en este caso su saber implica un saber de sí misma, modificará su concepción de sí misma y, por tanto, a sí misma.

El primer cambio que sufre la autoconciencia a partir de esta determinación de la vida es incorporar las transformaciones que ha sufrido su objeto inmediato. La autoconciencia se vuelve vida y género, y cree "que ha llegado a ser de modo objetual $^{\prime 54}$. Aunque ella se concibe a sí misma -y se constituye- como autoconciencia viva y género, esto sólo ocurre bajo la forma de la certeza, no como ser efectivo.

El yo producto de la absorción del objeto vivo es caracterizado como: (1) certeza de sí, siendo él mismo su propio objeto; (2) deseo, afirmándose a sí mismo a través de la negación del objeto; (3) vida; (4) género. Así, (1) el objeto de la

\footnotetext{
${ }^{52}$ Loc. cit.

${ }^{53}$ Loc. cit.

${ }^{54}$ Loc. cit.
} 
autoconciencia es el propio yo, que sólo es en la reflexión y (2) alcanzado en la medida en que niega el objeto inmediato, y en consecuencia, volviendo a ser deseo anulador. Ahora bien, este yo simple se concibe y constituye como (3) vida y (4) género. El producto de la absorción del objeto vivo como para sí es una autoconciencia que se define como negatividad, pero también como vida y género.

La noción de género es clave para la meta final de esta sección. La autoconciencia tiene por objeto al yo como género. Esto significa tenerlo como tipo de objeto que admite multiplicidad de casos. La autoconciencia como mera unidad yo=yo jamás podría tener experiencia de otros yoes, pues la noción inmediata de yo está ineludiblemente referida sólo al propio yo. El caso individual satura plenamente el concepto, con lo cual, no habría experiencia de objeto (como diferente a la autoconciencia) adecuado al saber. Ahora bien, comprender al yo como género permite reconocer objetos como yoes que no son yo mismo, tener experiencia de múltiples objetos autoconscientes sin confundir estas otras autoconciencias con la propia. Esta experiencia sería la realización de la certeza de la autoconciencia como esencia.

El emerger de otras autoconciencias como objeto de conocimiento de la autoconciencia es el modo de referir su diferencia a su unidad. Esta identificación sólo podrá realizarse en la experiencia de los múltiples objetos de la experiencia como autoconciencias. Así, aquello otro que sólo sería un mero objeto negado se presentará como saber, tal como la autoconciencia se concibe a sí misma (y con la consecuente posibilidad de que ella sea efectivamente en el ser y no en la mera reflexión).

En la experiencia que seguirá a lo largo de los restantes capítulos de la "Autoconciencia" "se le enriquecerá este objeto abstracto [puro yo], manteniéndose el despliegue que hemos visto en la vida" ${ }^{55}$. Los pasos a seguir de la "Autoconciencia" consisten en una sucesiva determinación del objeto "yo" de la autoconciencia, una experiencia de los otros yoes, y la consecuente conversión de la propia figura de la autoconciencia. Con la experiencia de otros yoes, se da un paso fundamental en la

\footnotetext{
${ }^{55}$ Loc. cit.
} 
consecución de los objetivos generales de la Fenomenología, pues por primera vez se presenta para el saber un objeto que se define como idéntico a él.

Ahora bien, para que la figura de la autoconciencia se realice efectivamente, su objeto debería presentársele como siendo, no como "saber". El objeto debe presentarse como la esencia del saber, esto es, en concordancia con tal saber, pero a modo de su objeto. En consecuencia, la realización efectiva de esta figura supone que el saber no se limite a tenerse como un saber que niega a su objeto (que se sabe saber y que reconduce el objeto a mero saber de la conciencia). Tal realización exige que el saber se convierta efectivamente en ser: que se presente como un objeto sapiente. En tanto la autoconciencia no supere su figura como deseo (es decir, como fuerza negadora del objeto), la esencia (lo objetivo) no se presentará como autoconciencia y, en consecuencia, no se realizará su verdad. Y, consecuentemente, ella no se realizará en el plano del ser.

Paradógicamente, esta realización se alcanza a través de la autonomía y resurgimiento del objeto de la conciencia. En primer lugar, este objeto resurge como una necesidad de la conciencia. Sin el polo objetivo por negar, la conciencia como deseo no puede conformar su identidad. Pero, en el estadio de la autoconciencia, el resurgimiento del objeto es el emerger de lo autoconsciente en el polo objetivo, realizando el saber de la autoconciencia. En tanto la autoconciencia exige la negación del objeto -pero sin poder llevarla a cabo porque le resulta esencial- y éste se presenta como autónomo, es menester que su negación sea hecha en el objeto mismo, que el objeto en sí mismo sea tal negación, lo que lo convierte en conciencia.

En otras palabras, si el objeto se desarrolla y se determina como autónomo, debe ser también una fuerza negadora, una estructura reflexiva que niegue su otredad y vuelva a sí mismo. Es el propio polo objetivo el que debe ejecutar su negación. Con ello, la negación característica de la autoconciencia emerge en el objeto convirtiéndolo en autoconciencia. Sólo resta dar el último paso que revelará que "la autoconciencia alcanza su satisfacción sólo en otra autoconciencia” ${ }^{56}$.

\footnotetext{
${ }^{56}$ Op. Cit. HEGEL, Fenomenología del espíritu, p. 255.
} 
Consideremos una esquematización del movimiento que ha sufrido la autoconciencia. En primer lugar, el mero yo puro, objeto pretendidamente inmediato de la autoconciencia. En segundo lugar, la necesidad de la anulación del objeto, por medio de lo cual el yo deja de ser el objeto inmediato de la reflexión y se descubre como mediado por la negación del polo objetivo. Esta figura es caracterizada como deseo. Aquí aun no se realiza la autoconciencia en tanto permanece en una reflexión interna, tomando su saber por objeto. En el tercer momento, "la duplicación de la autoconciencia" es "la verdad de esta certeza" ${ }^{57}$. Se presenta el objeto concordante con este saber, con lo que "hay un objeto para la autoconciencia que, en sí mismo, pone su ser otro, o la diferencia, como un objeto nulo y es autónomo al hacerlo" y es "género para sí mismo, [...]; es autoconciencia viviente" ${ }^{58}$. Así, el objeto vivo autónomo, a través de una negación interna propia, se vuelve autoconciencia viva, siendo así la realización efectiva (en el ser mismo) de la certeza alcanzada por la autoconciencia. El saber de sí misma de la autoconciencia y de su objeto como yo abstracto, como mera certeza verdadera, ahora deviene objeto y se realiza efectivamente en el ser. La autoconciencia no sólo tiene experiencia del objeto concordante con su saber de sí misma, sino que ella misma finalmente es efectivamente autoconciencia viva. Con la constitución del ser como vida autoconsciente la autoconciencia se realiza ella misma en el ser como vida.

Por esto, Hegel afirma que recién aquí este objeto es "una autoconciencia para una autoconciencia" ${ }^{\prime 59}$. La unidad buscada desde las primeras páginas del presente capítulo comienza a encontrarse; por primera vez, la autoconciencia tiene un objeto en el que puede reconocerse, satisfaciendo así su unidad con aquello otro de sí puesto por ella. Este objeto es un yo.

\section{Conclusiones}

La Fenomenología del espíritu persigue la absoluta identidad de ser y pensar a través de la sucesiva eliminación de todo objeto presuntamente autónomo al pensar.

\footnotetext{
${ }^{57}$ Loc. cit.

${ }^{58}$ Loc. cit.

${ }^{59}$ Loc. cit.
} 
La "Autoconciencia" da un paso clave hacia esta meta en tanto permite alcanzar una forma del saber que posibilita el primer surgimiento de un objeto sapiente. Esto supone una primera identificación profunda entre ser y pensar. En el enriquecimiento que sufre la conciencia y su objeto a través del carácter dialéctico de la experiencia se consigue una equiparación entre saber y ser que no deja resto incognoscible en el objeto del conocimiento, superando el dualismo kantiano, pero convirtiendo a su vez al ser mismo en saber. En este sentido, la "Autoconciencia" muestra cómo objeto y saber conforman una nueva unidad en la que el saber adquiere su realización y el objeto el pensamiento. Por su parte, esta meta y esta caracterización del objeto del conocimiento (experiencia) impiden la utilización de argumentos trascendentales. Pues, tal metodología sólo sería viable frente a una experiencia estática. La propuesta metodológica hegeliana supone una experiencia cambiante en concordancia con los cambios sufridos por la conciencia cognoscente.

Como hemos podido apreciar, el surgimiento de la autoconciencia en la Fenomenología del espíritu no puede explicarse como un reiterado retroceso lógico hacia las condiciones de posibilidad de una experiencia estática, ya constituida, y en consecuente la Fenomenología no puede ser entendida como el desarrollo de un argumento trascendental extenso. Entre las diferencias fundamentales entre Kant y Hegel, hemos destacado el carácter dinámico de la experiencia, esto es, el carácter dinámico del objeto en su constitución: los cambios epistemológicos del sujeto cognoscente suponen un cambio en su estructura categorial y el consecuente cambio de la experiencia misma. Esta diferencia radical en la consideración de la experienica y de la estructura categorial del sujeto cognoscente conduce a rechazar una lectura de la Fenomenología que pretenda hacer de ella una mera mejora de la deducción trascendental kantiana, ejecutada con el mismo método o los mismos objetivos con los que Kant ideó tal parte de la "analítica trascendental". En conclusión, al considerar el surgimiento de los otros yoes, al advertir el carácter dinámico de los objetos de la experiencia, advertimos un componente nuevo, una innovación de Hegel que hace imposible traducirla a términos metodológicos kantianos como pareciera ser la intención de Pippin. 


\section{Bibliografía}

- BEISER, Frederick, The Fate of Reason. German Philosophy from Kant to Fichte, Hardvard University Press, Cambridge, 1993.

- COLOMER, Eusebi, El pensamiento alemán de Kant a Heidegger, T. II El Idealismo: Fichte, Schelling y Hegel, Herder, Barcelona, 2006.

Idealismo: Fichte, Schelling y Hegel, Herder, Barcelona, 2006

- DE BOER, Karin, "The dissolving force of the concept: Hegel's ontological logic", The Review of Metaphysics, Vol. 57, No. 4, Jun., 2004

- --------------, On Hegel. The sway of the negative, Palgrave Macmillan, Hampshire, 2010

- DI GIOVANNI, George, HARRIS, H. S., Between Kant and Hegel. Texts in the Development of Post-Kantian Idealism, Hackett Publishing Company, Indianapolis/Cambridge, 2000. AMERIKS, Karl, Kant and the Fate of Autonomy. Problems in the Appropriation of the Critical Philosophy, Cambridge University Press, Cambridge, 2000.

- FABRO, Cornelio, La Dialéctica de Hegel, Editorial Columba, Bs. As., 1969,

- HEGEL, G.W.F Ciencia de la lógica, Ed. Solar, Bs. As, Trs. A. y R. Mondolfo, 1976

- - -----------., Fenomenología del espíritu, Madrid, ABADA / UAM Editores, ed. bilingüe de Antonio Gómez Ramos, 2010

- HONNETH, Axel. La lucha por el reconocimiento. Por una gramática moral de los conflictos sociales, Ed. Crítica Grijalbo Mondadori, Barcelona, 1997

- HOULGATE, Stephen, "Thought and Being in Kant and Hegel," The Owl of Minerva, 22, no. 21991

- -----------------, The opening of Hegel's Logic, Purdue University Press, Indiana, 2006

- HOYOS, Luis Eduardo, El escepticismo y la filosofía trascendental. Estudios sobre el pensamiento alemán a fines del siglo XVIII, Siglo del Hombre Editores, Bogotá, 2001 
- HYPPOLITE, J., Génesis y estructura de la Fenomenología del espíritu, Ediciones 62, Barcelona, 1991

-

- WESTPHAL, Kenneth. Hegel's epistemological realism. A Study of the aim and method of Hegel's Phenomenology of spirit, Kluwer Academic Publishers, Dordrecht, 1989

- KOJÉVE, Alexandre. La dialéctica del amo y del esclavo en Hegel, Fausto, Buenos Aires, 1996

- KREINES, James, "Between the Bounds of Experience and Divine Intuition: Kant's Epistemic Limits and Hegel's Ambitions", Inquiry, vol. 50, № 3, junio, 2007

- LABARRIERE, Pierre-Jean, La Fenomenología del Espíritu de Hegel. Introducción a una lectura, Fondo de Cultura Económica, México, 1979

- LONGUENESSE, Béatrice, Hegel's Critique of Metaphysics, Cambridge, Cambridge University Press, 2007

- PIPPIN, Robert. Hegel on Self-consciousness. Desire and Death in the Phenomenology of Spirit, Princeton University Press, New Jersey, 2011

- ------------ .Hegel's Idealism. The satisfactions of self-consciousness, New York, Cambridge University Press, 1989.

- WILLIAMS, Robert R., "Hegel and Transcendental Philosophy", en The Journal of Philosophy, Vol. 82, No. 11 (Nov., 1985) 